\title{
A Markov Approach to Exchange Rate Sentiment Analysis of Major Global Currencies
}

\author{
Kuthyar Shesha Madhava Rao ${ }^{1^{*}}$, Anjana Ramachandran ${ }^{2}$ \\ ${ }^{1}$ Department of Statistics, University of Botswana, Gaborone, Botswana \\ ${ }^{2}$ Department of Mathematics, University of Botswana, Gaborone, Botswana \\ Email: ${ }^{*}$ raom@mopipi.ub.bw
}

How to cite this paper: Rao, K.S.M. and Ramachandran, A. (2016) A Markov Approach to Exchange Rate Sentiment Analysis of Major Global Currencies. Open Journal of Statistics, 6, 1181-1195. http://dx.doi.org/10.4236/ojs.2016.66096

Received: August 16, 2016

Accepted: December 27, 2016

Published: December 30, 2016

Copyright $\odot 2016$ by authors and Scientific Research Publishing Inc. This work is licensed under the Creative Commons Attribution International License (CC BY 4.0). http://creativecommons.org/licenses/by/4.0/

\begin{abstract}
The paper deals with the analysis of exchange rates sentiments. In the approach suggested here a typical exchange rate sentiment is defined on the basis of certain function of mean and standard error of the logarithm of the ratio of successive daily exchange rates. Based on this surmise, the exchange rate sentiments are classified into various states, whereby states are named according to the perceptions of the market player. A Markov model is built to capture the uncertainties in exchange rates sentiments. The approach advocated here will be of interest to researchers, exchange rate traders and financial analysts. As an application of the proposed line of approach, we analyse weekly and monthly exchange rate sentiments that govern exchange rates of the major global currencies-EUR, GBP, SDR, YEN, ZAR, USD, using data from 2001-2012. Some interesting conclusions are revealed based on the data analysis approach advocated in this paper.
\end{abstract}

\section{Keywords}

Ergodicity, Exchange Rates Sentiments, Markov Model, Transition Probability, Weekly and Monthly States

\section{Introduction}

The analysis of stochastic volatility models is vital due to its applications in the areas of high frequency finance. In a statistical perspective, methods for fitting stochastic volatility (SV) models have the potential for wider applications. But applications for the modeling and prediction of time-varying volatility on financial markets cannot be undermined. These models are fundamental tools in risk management, asset pricing and asset allocation. The appropriate fitting of models with stochastic volatility is one of the demanding problems in time series analysis. 
Stochastic volatility models can be interpreted as non-linear state space models in which the unobserved state is the stochastically evolving log volatility of the process. Since data is characteristically observable only at discrete points in time, discrete-time formulations of SV models are important in empirical applications. In financial mathematics and financial economics, stochastic volatility is typically modeled in a continuous-time setting. This formulation proves beneficial for derivative pricing and portfolio optimization. Constant volatility models have been shown to provide an inferior fit of financial time series. A more practical approach to volatility modeling is provided by dynamic structures. Stochastic volatility models are propellers for the modeling and prediction of time-varying volatility on financial markets.

Derivative pricing and portfolio optimization have benefitted from continuous-time stochastic volatility models and thus have figured prominently in financial mathematics and financial economics. The data being only observable at discrete points in time, discrete-time formulations of SV models are essential. Estimation of volatility, the wide swings observed in daily, weekly or monthly exchange rates can be studied, by convention, using discrete-time process. The arbitrary shift of volatility of exchange rates from one state to another in a random fashion is the corner stone of the approach advocated in this paper.

\section{Literature Review}

One approach to modeling time-varying volatility comes from the stochastic volatility models. The mixture-of-distribution hypothesis $(\mathrm{MDH})$ postulated by Clark [1], efficiently supports SV models. According to this hypothesis, asset returns follow a mixture of normal distributions with a mixing process depending on the (unobservable) information arrival process. A characteristic feature of financial return series is revealed for a positively auto correlated mixing process, namely volatility clustering. The $\mathrm{MDH}$ proposed that asset return volatility follows its own stochastic process which is restructured by unobservable developments, in contrast to the other approach, an autoregressive conditional heteroscedasticity $(\mathrm{ARCH})$ model introduced by Engle [2], followed by its generalization (GARCH) by Bollerslev [3] and other dynamic versions. Here, given the available information set, the conditional variance is a function of past observations.

The two approaches differ in the structure of the volatility process. The former follows its own stochastic process, with potential dependence coming through the correlation structure between the series and the volatility processes while the latter assumes dependence on the previous realized shocks in the series.

The likelihood of SV models is not directly available making it more complex compared to the widely used (Generalized) ARCH model. This SV framework requires the use of simulation techniques, like simulated maximum likelihood, method of simulated moments or Markov Chain Monte Carlo (MCMC) techniques. Computational costs render SV models less popular in financial practice although increasing computer efficiency and progress of competent sampling techniques weaken this drawback to a great 
extent. The basic SV model has been found to be restrictive for many financial time series. In this context, the relationship between volatility and price/return has been studied extensively.

SV models provide an ideal framework to accommodate specific properties of financial return processes such as fat-tailedness, leverage effects and the occurrence of jumps.

Estimation of stochastic volatility models has been widely studied. Generalizations of these models include jumps in returns and in volatilities and/or fat tails in distributions. Extensions include Bayesian analysis of univariate stochastic volatility (SV) processes, as in Jacquier et al., [4] and multivariate stochastic volatility models proposed by Harvey et al., [5].

Previous study by Rebonato [6] assumed that the instantaneous volatility of the forward rates was a deterministic function of time to maturity only. A model of the swaption market that consisted of a hidden Markov chain with a normal and excited state was the focus of this study. The original deterministic volatility model does not accurately describe a market that is in a temporary "excited" state. The study by White et al. [7] extended this model in which two volatility curves exist: an excited curve and a normal curve for the instantaneous forward volatility. The volatilities in the swaption matrix result from averaging over all possible paths along the Markov chain. At any given time, the market was in one of these states and the transitions between these states were governed by a hidden Markov chain. Previous studies have used Markov chain models to describe option prices, but this study in interest rates involved transitions between functions rather than level. This model was applied to a longer period of US dollars at-the-money swaption volatility market data.

The study by Hautsch et al. [8] reviewed the general framework of discrete-time stochastic volatility models (SV) and illustrated the major principles of corresponding Markov Chain Monte Carlo (MCMC) based statistical inference. A practical approach was provided and that was implemented in empirical applications and financial practice. The results were based on different SV specifications using returns on stock indices and foreign exchange rates. The model was unable to capture the distributional properties of the returns.

Park [9] investigated the relationship between volatility and herd behaviour, a mounting topic in financial economics. The study developed a herd-behaviour-stochastic-volatility model that explained herding in financial markets and considered a Markov Chain Monte Carlo method as a competent method for estimating the model. The study illustrated theoretically that herd behaviour lead to a high increase in volatility but not trading volume as opposed to information inputs that increased in both volatility and trading volume. Empirical application with high frequency data in the Korean equity market supported this theory. The study proposed a methodology for detecting trading days with herding. This was based on the theoretical results from the percolation model that was based on the hypothesis of information cascades due to flawed information and the concepts of realized volatility and realized bipolar variation.

Hahn et al. [10] estimated the parameters governing a continuous time Markov 
switching model with observations given at discrete times only. The states for drift, volatility and the generator matrix of the underlying Markov process based on discretely observed data were estimated.

The extent of liquidity risk due to co-movements was studied within each class of market capitalization by Beaupain et al. [11]. The analysis of liquidity co-movements was dependent on volatility regime states. On an average, the extent of liquidity co-movements was positively related to the market capitalization of the portfolio. Also liquidity co-movements were least intense among small caps and most intense among large caps. On a cross-sectional basis, the extent of class-wide liquidity co-movements was typically greater in quiet markets for both large and mid-caps.

Chun et al. [12] proposed a method for calibrating the local volatility surface that reduced the computational complexity associated with many models and price options with the volatility skew. A continuous time semi-closed form solution based on the Markov chain approximation of Kushner [13] was adopted which could explain diffusion or a jump-diffusion. The study showed that the out-of-sample options can be accurately priced for both cases without regularisation.

The superiority of MRS-GARCH models to GARCH type of models in terms of modelling and forecasting gold price volatility was investigated by Sopipan et al. [14]. The study forecast the volatility of gold prices using Markov Regime Switching GARCH (MRS-GARCH) models. The models allow volatility to follow different dynamics according to unobserved regime variables. The closing prices of gold to trade future contract were predicted.

In this paper we advocate a new approach based on the market sentiments to analyse financial data rather than the traditional approach based on volatility measures. The analysis of financial data based on the market sentiments rather than the volatility has been recently advocated by Rao and Moseki [15]. Rao and Moseki, defined a typical market sentiment on the basis of certain function of mean and standard error of the Domestic Company Indices of Botswana Stock Exchange. Based on this surmise, the market sentiments were classified into various states, whereby states were named according to the perceptions of the market player. A multinomial probability model was built to capture the uncertainties in market sentiments. Two asymptotically distribution-free tests, namely the chi-square and the likelihood ratio test of goodness of fit for the hypothesis of the symmetry in market sentiments were suggested. Later on, Rao and Moseki [16] advocated an alternative approach, namely, a Markov model approach to analyse stock market sentiments based on the constructs detailed in [15]. They examined stationarity of the transition probability matrix associated with the market sentiments in DCI of BSE. Following the works of [15], Rao and Anjana [17] analysed market sentiments of exchange rates of major global currencies. The data considered in their analysis referred to the logarithm of the ratio of successive daily exchange rates as opposed to the daily DCI considered in [16].

The objective of this paper is to apply Markov approach of [16] to analyse market sentiments associated with major global currencies. The approach advocated here will 
be of interest to researchers, exchange rate traders and financial analysts. Section 2, describes a Markov model for market sentiments. The section deals with a certain classification rule that generates market sentiment states for the exchange rate data. The method of construction of a transition probability matrix and derivation of steady state probabilities are also discussed. The application of the proposed model and the related weekly and monthly market sentiment analysis pertaining to exchange rate of major global currencies is carried out in Section 3. The exchange rates data were drawn from the Bank of Botswana's Botswana Financial Statistics database. The six currencies considered in the analysis are Euro (EUR), British Pound (GBP), Special Drawing Rights (SDR), Japanese Yen (YEN), South African Rand (ZAR) and US Dollar (USD). All the exchange rates have been quoted in terms of Botswana Pula (BWP). The time series data refers to the working day exchange rates of the six stated currencies for 12 years starting from 2001 and ending with 2012. Section 4 concludes the paper.

\section{A Markov Model for Exchange Rate Sentiments}

In this section we develop the approach which lead to the construction of market sentiment states from the given time series data of exchange rate. From the so obtained sequence of exchange rate market sentiment states, we build a Markov Model which captures the movement of exchange rate phenomena in terms of the transition probabilities among the exchange rate states. Using the estimated transition probability matrix (TPM), we explore the behaviour of market sentiment states.

\subsection{A Seven State Exchange Rate Sentiment Classification Rule}

Suppose that the time series data $X_{\ell}, \ell=1, \cdots, N$, related to exchange rates of major global currencies for period of $N$ working days is available on a day to day basis. As in Rao and Anjana [17], we use the following notations and the associated classification rule: " $N$ " - total number of days in a study period, " $\ell$ "-refers to the day, " $W$ " - total number of weeks in a study period, " $w$ "-refers to the week, " $n_{w}$ "-denotes the number of business days in a week. Here, $n_{w}$ varies between 1 and 5 in a typical FOREX market. The data considered for the analysis here refer to the logarithm of the ratio of the day's exchange rate $\left(X_{\ell}\right)$, with the previous day's exchange rate $\left(X_{\ell-1}\right)$, i.e., log return defined by

$$
Y_{\ell}=\ln \left(\frac{X_{\ell}}{X_{\ell-1}}\right), \ell=2, \cdots, N .
$$

Let the average $\bar{Y}_{w}$ of $Y_{\ell}^{w}$, for the week $w$ be given by

$$
\bar{Y}_{w}=\frac{1}{n_{w}} \sum_{\ell=1}^{n_{w}} Y_{\ell}^{w}, w=1, \cdots, W,
$$

and the variance $s_{w}^{2}$ of $Y_{\ell}^{w}$, for the week $w$ be given by

$$
s_{w}^{2}=\frac{1}{n_{w}-1} \sum_{t=1}^{n_{w}}\left(Y_{t}^{w}-\bar{Y}_{w}\right)^{2} .
$$


For the week $(w+1), w=1, \cdots, W-1$, the weekly market sentiment of exchange rate is said to belong to

1) State $S_{1}$, if $\bar{Y}_{w+1}<\bar{Y}_{w}-3 \frac{S_{w}}{\sqrt{n_{w}}}$

2) State $S_{2}$, if $\bar{Y}_{w}-3 \frac{s_{w}}{\sqrt{n_{w}}} \leq \bar{Y}_{w+1}<\bar{Y}_{w}-2 \frac{s_{w}}{\sqrt{n_{w}}}$

3) State $S_{3}$, if $\bar{Y}_{w}-2 \frac{s_{w}}{\sqrt{n_{w}}} \leq \bar{Y}_{w+1}<\bar{Y}_{w}-\frac{s_{w}}{\sqrt{n_{w}}}$

4) State $S_{4}$, if $\bar{Y}_{w}-\frac{s_{w}}{\sqrt{n_{w}}} \leq \bar{Y}_{w+1}<\bar{Y}_{w}+\frac{s_{w}}{\sqrt{n_{w}}}$

5) State $S_{5}$, if $\bar{Y}_{w}+\frac{s_{w}}{\sqrt{n_{w}}} \leq \bar{Y}_{w+1}<\bar{Y}_{w}+2 \frac{s_{w}}{\sqrt{n_{w}}}$

6) State $S_{6}$, if $\bar{Y}_{w}+2 \frac{s_{w}}{\sqrt{n_{w}}} \leq \bar{Y}_{w+1}<\bar{Y}_{w}+3 \frac{s_{w}}{\sqrt{n_{w}}}$

7) State $S_{7}$, if $\bar{Y}_{w+1}>\bar{Y}_{w}+3 \frac{S_{w}}{\sqrt{n_{w}}}$.

Rao and Anjana [17] named these seven states respectively as: the dread sentiment $\left(S_{1}\right)$, the bearish sentiment $\left(S_{2}\right)$, the decline sentiment $\left(S_{3}\right)$, the calm sentiment $\left(S_{4}\right)$, the incline sentiment $\left(S_{5}\right)$, the greed sentiment $\left(S_{6}\right)$, the bullish sentiment $\left(S_{7}\right)$. It may be pointed out that states $S_{5}, S_{6}$ and $S_{7}$ reflect smiling sentiments with varying degree while, states $S_{1}, S_{2}$ and $S_{3}$ reflect frowning sentiments with varying degree.

\subsection{A $k$-State Sentiment Model for Exchange Rates}

We assume that volatility in foreign exchange rates can be described by a Markov-like model so that the weekly and monthly market sentiments can be classified into seven states based on the classification rule (3) discussed in the previous section. The assumption is valid since the weekly market sentiments constructs are designed such that immediate future market sentiment construct depends only on the current state construct and not on the past states. Suppose that for a given $\log$ return data $Y_{\ell}, \ell=2, \cdots, N$, we have the generated weekly market sentiment states $\left\{S_{u, v}, v \in(1,7), u=2, \cdots, W\right\}$ obtained according to the classification rule (3). We briefly indicate below the theory related to the exposition of properties of a finite Markov Chain and inferences related to the TPM of the Markov Chain. In general, given the $k$ states, let

$n_{i j}, i=1, \cdots, k ; j=1, \cdots, k$, denote the number of transitions from state $i$ to $j$. Let $\sum_{i j} n_{i j}=n_{i}$. Let $p_{i j}, i, j=1,2, \cdots, k$ be the one-step transition probability from the state $i$ to state $j$ and the TPM of the Markov Chain be given by

$$
P=\left(p_{i j}\right)=\left(\begin{array}{cccc}
p_{11} & p_{12} & \cdots & p_{1 k} \\
p_{21} & p_{22} & \cdots & p_{2 k} \\
\vdots & \vdots & \ddots & \vdots \\
p_{k 1} & p_{k 2} & \cdots & p_{k k}
\end{array}\right)
$$


For a given initial state $i$ and a number of trials $n_{i}$, the sample of transition counts $\left(n_{i 1}, n_{i 2}, \cdots, n_{i k}\right)$ can be considered as a sample of size $n_{i}$ from a multinomial distribution with probabilities $\left(p_{i 1}, p_{i 2}, \cdots, p_{i k}\right)$, such that $0<p_{i j}<1, \sum_{i=1}^{k} p_{i j}=1$. Then it is well known that (refer to Bhat [18]) the maximum likelihood estimators of $p_{i j}$ are given by

$$
\hat{p}_{i j}=\frac{n_{i j}}{n_{i}}, i, j=1,2, \cdots, k .
$$

Once we estimate the transition probability matrix $P$ by $\hat{P}=\left(\hat{p}_{i j}\right)$ for the weekly market sentiments of foreign exchange rates, we can explore the salient features of the exchange rate sentiment states in terms of the properties of the finite Markov Chain $\hat{P}$. For example, it would be interesting to 1) classify the sentiment states 2) find the stationary distribution of states and 3) compute the mean recurrence time for each state. Suppose that the limits $\pi_{i}=\lim _{n \rightarrow \infty} p_{j i}^{(n)}$ exist such that $\pi_{i} \geq 0, \sum \pi_{i}=1$ and are independent of the initial state $j$ for an irreducible ergodic chain. Then the limiting probability distribution $\left\{\pi_{i}\right\}$ is identical to the stationary distribution of the given chain. We recall that the stationary distribution of the Markov chain is given by

$$
\pi_{i}=\sum_{j=1}^{k} \pi_{j} p_{j i}, \sum_{i=1}^{k} \pi_{i}=1, i=1, \cdots, k .
$$

Finally the expected value of the recurrence time for state " $p$ " is given by

$$
\tau_{i i}=\frac{1}{\pi_{i}}, i=1,2, \cdots, k .
$$

It may be pointed out that the theoretical discussion of the aforementioned concepts can be found in Isaacson \& Madsen [19] and we shall give the necessary computations in the example to be discussed in section 3 below. Further, a second problem of interest would be to investigate whether the weekly or monthly exchange rate market sentiments are stationary over a certain study period say $T(>1)$. Such a question can be addressed in terms of the estimation and associated hypothesis testing problem applied to finite Markov chains with stationary (time-homogeneous) transition probability matrices as detailed below.

Suppose that the weekly TPM corresponding to the year " $t$ " be given by $p_{i j}^{t}, t=1,2, \cdots, T$. Then the assumption that the weekly market sentiments over the " $T$ " years are stationary is equivalent to test of the null hypothesis $H_{0}: p_{i j}^{t}=p_{i j},(t=1,2, \cdots, T)$. A likelihood ratio test statistic for testing $H_{0}$, the stationarity of the TPM, can be developed as follows:

Let $p_{i j}^{t}$ be the one-step transition probability of a time-dependent Makov process $X(t)$, such that

$$
p_{i j}^{t}=P[X(t+1)=j \mid X(t)=i]
$$

Let $n_{i j}^{t}$ be the number of transition from " $i$ " to " $j$ " $(i \rightarrow j)$ during the $t$-th transition of a process $i=1, \cdots, k, j=1, \cdots, k, t=1, \cdots, T$. The maximum likelihood estimates of $p_{i j}^{t}$ can be obtained as 


$$
\hat{p}_{i j}^{t}=\left(\frac{n_{i j}^{t}}{n_{i}^{t-1}}\right), \text { where, } n_{i}^{t-1}=\sum_{j=1}^{k} n_{i j}^{t} .
$$

Note that $n_{i}^{t-1}$ is the number of processes in state $i$ at time $(t-1)$. To test the null hypothesis $H_{0}: p_{i j}^{t}=p_{i j}(t=1,2, \cdots, T)$ versus the alternative $H_{1}: p_{i j}^{t} \neq p_{i j}$ for at least one $t$, using the standard notations, the likelihood ratio criterion is given by

$$
\Lambda=\frac{L\left(\hat{p}_{i j}^{t}\right)}{L\left(\hat{p}_{i j}\right)} .
$$

Using straight forward simplifications it can be shown that,

$$
-2 \ln \Lambda=2 \sum_{t=1}^{T} \sum_{i=1}^{k} \sum_{j=1}^{k} n_{i j}^{t} \ln \left(\frac{n_{i j}^{t}}{n_{i}^{t-1} p_{i j}}\right)
$$

and under the null hypothesis $H_{0},-2 \ln \Lambda$ has a Chi-Square distribution with $(T-1)[k(k-1)]$ degrees of freedom. If some of the $n_{i j}^{t}$ 's are zeroes, then the summation in (9) is taken only over those $(i, j)$ pairs for which $n_{i j}^{t}>0$. Then the degrees of freedom of $\chi^{2}$-statistics will be $(T-1)[k(k-1)]-d$, where " $d$ " is obtained by taking into account that some of the $n_{i j}^{t}$ 's are zero.

\section{Applications to Major Global Currency Exchange Rate Sentiments}

The application of the proposed model and the analysis of related weekly and monthly exchange rate is considered in this section. The daily FOREX data were drawn from the Bank of Botswana's Botswana Financial Statistics database. The six currencies considered for the purpose of analysis are Euro (EUR), Great Britain Pound (GBP), Special Drawing Rights (SDR), Japanese Yen (YEN), South African Rand (ZAR) and United States Dollar (USD). The data considered is for a 12 year period from January 2001 to December 2012. The computations leading to weekly and monthly log returns, classification of the log returns to the exchange rate sentiment states according to the rule (3), derivation of steady state distributions and likelihood ratio test are not reported in this paper; but readers interested in these elaborate computations may contact the first author. Given time series of exchange rates consisting of $W$ weeks of the year $t=1, \cdots, T$, we compute $(W-1)$ transitions of exchange rate sentiment states using the rule (3) described in section 2.1. Let $n_{i, j}, i, j=1, \cdots, 7$ be the number of transitions from state " $i$ " to state " $j$ " and $n_{i}=\sum_{j=1}^{k} n_{i j}$ be the total number of times the process visits the state " $i$ ", $i=1, \cdots, 7$. for a given year " $t$ ". Note that $\sum_{j=1}^{7} n_{i}=(W-2)$. Then the maximum likelihood estimator of transition probabilities from state “" to state " $j$ " is given by $\hat{p}_{i j}=\frac{n_{i j}}{n_{i}}$.

\subsection{Analysis of Weekly Exchange Rate Sentiments}

The application of the proposed model and the related weekly and monthly analysis 
pertaining to FOREX rate is carried out in this section. The time series data refers to the working day exchange rates of the six stated currencies for 12 years starting from January of 2001 and ending with December of 2012. The data considered for the analysis here refer to the logarithm of the ratio of the day's exchange rate $\left(X_{\ell}\right)$, with the previous day's exchange rate $\left(X_{\ell-1}\right)$, i.e., $Y_{\ell}=\ln \left(\frac{X_{\ell}}{X_{\ell-1}}\right), \ell=2, \cdots$. The data are clustered into 626 weeks, where each week consists of 5 working days, Monday through Friday. The movements in the exchange rates are classified into the seven states

$S_{1}, S_{2}, S_{3}, S_{4}, S_{5}, S_{6}, S_{7}$ based on the rule described by (3). The estimated transition probability matrix $\hat{P}=\left(\hat{p}_{i j}\right)$ for the weekly data using sequence of states generated by the classification rule (3) is calculated using the formula (5) described in section 2.2. We elaborate the computational procedure of obtaining TPM and the subsequent analysis of exchange rate sentiments for the currency EURO, while for other currencies; we simply provide the summary finding in the form of a table. For the period 2001 to 2012, the weekly FOREX transition count matrix and the estimated TPM for Euro is exhibited in Table 1 and Table 2. The entries in the Table 2 are calculated using the formula (5). For example,

$$
\hat{p}_{13}=\frac{2}{44}=0.045, \hat{p}_{26}=\frac{7}{31}=0.226, \hat{p}_{44}=\frac{37}{144}=0.257
$$

and so on.

Table 1. Weekly transition count matrix for EURO sentiments during 2001 to 2012.

\begin{tabular}{|c|c|c|c|c|c|c|c|c|}
\hline & Dread & Bearish & Decline & Calm & Incline & Bullish & Greed & No. of \\
\hline State & 1 & 2 & 3 & 4 & 5 & 6 & 7 & weeks \\
\hline Dread & 0 & 1 & 2 & 4 & 9 & 17 & 11 & 44 \\
\hline Bearish & 1 & 0 & 3 & 6 & 7 & 7 & 7 & 31 \\
\hline Decline & 1 & 2 & 4 & 12 & 20 & 22 & 24 & 85 \\
\hline Calm & 1 & 4 & 18 & 37 & 44 & 21 & 19 & 144 \\
\hline Incline & 16 & 11 & 27 & 44 & 34 & 15 & 7 & 154 \\
\hline Bullish & 13 & 3 & 17 & 23 & 22 & 5 & 7 & 90 \\
\hline \multirow[t]{2}{*}{ Greed } & 12 & 10 & 13 & 21 & 17 & 2 & 3 & 78 \\
\hline & & & & & & & & 626 \\
\hline
\end{tabular}

Table 2. Weekly TPM for EURO sentiments during 2001 to 2012.

$$
\hat{P}=\left(\hat{p}_{i j}\right)=\left(\begin{array}{lllllll}
0.000 & 0.023 & 0.045 & 0.091 & 0.205 & 0.386 & 0.250 \\
0.031 & 0.000 & 0.097 & 0.194 & 0.226 & 0.226 & 0.226 \\
0.012 & 0.024 & 0.047 & 0.141 & 0.235 & 0.259 & 0.282 \\
0.006 & 0.028 & 0.125 & 0.257 & 0.306 & 0.146 & 0.132 \\
0.105 & 0.071 & 0.175 & 0.286 & 0.221 & 0.097 & 0.045 \\
0.144 & 0.033 & 0.189 & 0.256 & 0.244 & 0.056 & 0.078 \\
0.154 & 0.128 & 0.167 & 0.269 & 0.218 & 0.026 & 0.038
\end{array}\right)
$$


Following standard computations, (see for example, Isaacson \& Madsen [19]), it can be shown that the transition probability matrix given by $\hat{P}$ is ergodic. Further, using the definition of ergodicity, it can be concluded that the Markov chain is 1) irreducible 2) persistently non-null and 3) aperiodic. The weekly expected recurrence time $\left\{\tau_{i i}, i=1, \cdots, 7\right\}$ for the seven states $S_{1}, \cdots, S_{7}$ are given by $\tau_{11}=14.31, \tau_{22}=20.24, \tau_{33}=7.46, \tau_{44}=4.26, \tau_{55}=4.09, \tau_{66}=7.04, \tau_{77}=8.03$.

The practical interpretation is that the EURO exchange rate bounced back to dread sentiment state $S_{1}$ once in every 14.31 weeks and to the incline sentiment $S_{5}$ once in every 4.09 weeks and so on, during the study period 2001 to 2012.

Turning to the test of the hypothesis of stationarity of weekly EURO FOREX sentiments during the study period 2001 to 2012, the application of the likelihood ratio test statistic given by (11) for Euro exchange rate yields $-2 \ln \Lambda=447.39$. Given the estimated TPM for the years 2001 to 2012, it can be shown that, under the alternative hypothesis, the number of parameters to be estimated is 243 , while under the null hypothesis, the parameters to be estimated is 40 . Thus the likelihood ratio test statistic has a chi-square distribution with 203 degrees of freedom. From the table of $\chi^{2}$ distribution, we get $P\left(\chi_{(203)}^{2} \geq 447.39\right)<0.005$. This implies that the hypothesis of stationarity of EURO FOREX sentiments during the period 2001 to 2012 cannot be accepted even with $0.5 \%$ significance level. In Table 3 , we provide the summary of stationary probabilities $(\pi)$ and expected recurrence times $\left(\tau=\frac{1}{\pi}\right)$ for all the six currencies considered. It is clearly seen from Table 3 that across the six exchange rates considered, the mean recurrence time for dread sentiment is around $14-21$ weeks, for calm sentiment

Table 3. Summary of weekly FOREX sentiments for the period 2001 to 2012.

\begin{tabular}{|c|c|c|c|c|c|c|c|c|}
\hline \multicolumn{2}{|c|}{ State } & \multirow{2}{*}{$\begin{array}{c}\text { Dread } \\
\pi_{1}\end{array}$} & \multirow{2}{*}{$\begin{array}{c}\text { Bearish } \\
\pi_{2}\end{array}$} & \multirow{2}{*}{$\begin{array}{c}\text { Decline } \\
\pi_{3}\end{array}$} & \multirow{2}{*}{$\begin{array}{c}\text { Calm } \\
\pi_{4}\end{array}$} & \multirow{2}{*}{$\begin{array}{c}\text { Incline } \\
\pi_{5}\end{array}$} & \multirow{2}{*}{$\begin{array}{c}\text { Bullish } \\
\pi_{6}\end{array}$} & \multirow{2}{*}{$\begin{array}{c}\text { Greed } \\
\pi_{7}\end{array}$} \\
\hline & & & & & & & & \\
\hline \multicolumn{9}{|l|}{ Currency } \\
\hline \multirow[t]{2}{*}{ EUR } & $\pi$ & 0.0699 & 0.0494 & 0.1341 & 0.2349 & 0.2447 & 0.1420 & 0.1245 \\
\hline & $\tau=1 / \pi$ & 14.3062 & 20.2429 & 7.4571 & 4.2571 & 4.0866 & 7.0423 & 8.0321 \\
\hline \multirow[t]{2}{*}{ GBP } & $\pi$ & 0.0622 & 0.0573 & 0.1518 & 0.2028 & 0.2701 & 0.1373 & 0.1186 \\
\hline & $\tau=1 / \pi$ & 16.0772 & 17.4520 & 6.5876 & 4.9310 & 3.7023 & 7.2833 & 8.4317 \\
\hline \multirow[t]{2}{*}{ SDR } & $\pi$ & 0.0654 & 0.0511 & 0.1342 & 0.2301 & 0.2651 & 0.1438 & 0.1099 \\
\hline & $\tau=1 / \pi$ & 15.2905 & 19.5695 & 7.4516 & 4.3459 & 3.7722 & 6.9541 & 9.0992 \\
\hline \multirow[t]{2}{*}{ YEN } & $\pi$ & 0.0606 & 0.0526 & 0.1548 & 0.2250 & 0.2346 & 0.1548 & 0.1165 \\
\hline & $\tau=1 / \pi$ & 16.5017 & 19.0114 & 6.4599 & 4.4444 & 4.2626 & 6.4599 & 8.5837 \\
\hline \multirow[t]{2}{*}{ ZAR } & $\pi$ & 0.0464 & 0.0799 & 0.1279 & 0.2558 & 0.2446 & 0.1279 & 0.1183 \\
\hline & $\tau=1 / \pi$ & 21.5517 & 12.5156 & 7.8186 & 3.9093 & 4.0883 & 7.8186 & 8.4531 \\
\hline \multirow[t]{2}{*}{ USD } & $\pi$ & 0.0590 & 0.0591 & 0.1198 & 0.2506 & 0.2635 & 0.1518 & 0.0969 \\
\hline & $\tau=1 / \pi$ & 16.9492 & 16.9205 & 8.3472 & 3.9904 & 3.7951 & 6.5876 & 10.3199 \\
\hline
\end{tabular}


it is around 4 - 5 weeks, for greed sentiment it is around 8 - 10 weeks and so on. Further, it is interesting to note that for all the FOREX currencies considered, mean recurrence time for states towards the dread sentiment is uniformly more than that of greed sentiment. Table 4 provides the summary of the likelihood ratio tests for the stationarity of FOREX sentiments. It is seen that the hypothesis of stationarity of exchange rate sentiments during the period 2001 to 2012 is rejected in all the cases. It is not surprising though, because of the fact that the period 2001 to 2012 witnessed a whole lot of economic crises such as US housing bubble which peaked in 2004, 2007-08 global economic crises triggered by US stock markets, European sovereign-debt crisis, among others.

\subsection{Analysis of Monthly Exchange Rate Sentiments}

In this section we proceed to analyse monthly exchange rate sentiments. The data are clustered into 144 months, where each month consists of 20 - 22 working days. We modify the formula (1), (2) and (3) for the monthly data and accordingly generate the transition count matrix and estimated TPM as displayed in Table 5 and Table 6.

As in section 3.2, based on the monthly data, stationary probability distribution $\left\{\pi_{i}, i=1, \cdots, 7\right\}$ corresponding to the Markov chain can be constructed applying the algebraic method. For the currency EURO, the stationary probabilities are estimated as follows.

Table 4. Summary of likelihood ratio test for stationarity of weekly FOREX sentiments.

\begin{tabular}{ccccc}
\hline Currency Observed value of LR statistics & Degrees of freedom Table value of chi square & Decision \\
\hline EUR & 447.3874 & 203 & 293.1762 & Reject null hypothesis \\
GBP & 477.6675 & 194 & 283.3283 & Reject null hypothesis \\
SDR & 422.5295 & 198 & 290.4230 & Reject null hypothesis \\
YEN & 436.9548 & 207 & 300.6157 & Reject null hypothesis \\
ZAR & 466.1922 & 192 & 279.0646 & Reject null hypothesis \\
USD & 435.2207 & 190 & 268.4680 & Reject null hypothesis \\
\hline
\end{tabular}

Table 5. Monthly transition count matrix for EURO sentiments during 2001 to 2012.

\begin{tabular}{ccccccccc}
\hline EUR & & & & & & & \multicolumn{2}{c}{ No. of } \\
\hline State & Dread & Bearish & Decline & Calm & Incline & Bullish & Greed & months \\
\hline Dread & 0 & 0 & 0 & 0 & 3 & 3 & 4 & 10 \\
Bearish & 0 & 0 & 0 & 2 & 2 & 3 & 2 & 9 \\
Decline & 0 & 0 & 2 & 3 & 5 & 7 & 2 & 19 \\
Calm & 1 & 1 & 4 & 5 & 9 & 8 & 2 & 30 \\
Incline & 2 & 1 & 7 & 9 & 8 & 6 & 1 & 34 \\
Bullish & 4 & 5 & 6 & 7 & 7 & 1 & 0 & 30 \\
Greed & 3 & 2 & 0 & 5 & 0 & 1 & 0 & 11 \\
& & & & & & & & 143 \\
\hline
\end{tabular}


Table 6. Monthly TPM for EURO sentiments during 2001 to 2012.

$\hat{P}=\left(\hat{p}_{i j}\right)=\left(\begin{array}{ccccccc}0.0 & 0.0 & 0.0 & 0.0 & 0.300 & 0.300 & 0.400 \\ 0.0 & 0.0 & 0.0 & 0.222 & 0.222 & 0.334 & 0.222 \\ 0.0 & 0.0 & 0.105 & 0.158 & 0.263 & 0.366 & 0.105 \\ 0.033 & 0.033 & 0.133 & 0.167 & 0.300 & 0.267 & 0.067 \\ 0.058 & 0.029 & 0.207 & 0.265 & 0.235 & 0.177 & 0.029 \\ 0.133 & 0.168 & 0.200 & 0.233 & 0.233 & 0.033 & 0.0 \\ 0.273 & 0.182 & 0.0 & 0.455 & 0.0 & 0.090 & 0.0\end{array}\right)$

Table 7. Summary of monthly FOREX sentiments for the period 2001 to 2012.

\begin{tabular}{ccccccccc}
\hline State & & Dread & Bearish & Decline & Calm & Incline & Bullish & Greed \\
\hline Currency & & $\pi_{1}$ & $\pi_{2}$ & $\pi_{3}$ & $\pi_{4}$ & $\pi_{5}$ & $\pi_{6}$ & $\pi_{7}$ \\
\hline EUR & $\pi$ & 0.0694 & 0.0622 & 0.1326 & 0.2164 & 0.2381 & 0.2039 & 0.0770 \\
& $\tau=1 / \pi$ & 14.4092 & 16.0772 & 7.5415 & 4.6211 & 4.1999 & 4.9044 & 12.9870 \\
GBP & $\pi$ & 0.0486 & 0.0765 & 0.1116 & 0.2805 & 0.2309 & 0.1603 & 0.0913 \\
& $\tau=1 / \pi$ & 20.5761 & 13.0719 & 8.9606 & 3.5651 & 4.3309 & 6.2383 & 10.9529 \\
SDR & $\pi$ & 0.0559 & 0.0559 & 0.1608 & 0.1748 & 0.3076 & 0.1747 & 0.0699 \\
& $\tau=1 / \pi$ & 17.8891 & 17.8891 & 6.2189 & 5.7208 & 3.2510 & 5.7241 & 14.3062 \\
YEN & $\pi$ & 0.0212 & 0.0836 & 0.1400 & 0.2649 & 0.2519 & 0.1680 & 0.0704 \\
& $\tau=1 / \pi$ & 47.1698 & 11.9617 & 7.1429 & 3.7750 & 3.9698 & 5.9524 & 14.2045 \\
\multirow{2}{*}{ ZAR } & $\pi$ & 0.0346 & 0.0349 & 0.1762 & 0.3157 & 0.1809 & 0.1466 & 0.1108 \\
& $\tau=1 / \pi$ & 28.9017 & 28.6533 & 5.6754 & 3.1676 & 5.5279 & 6.8213 & 9.0253 \\
\multirow{2}{*}{ USD } & $\pi$ & 0.0274 & 0.0903 & 0.1530 & 0.2233 & 0.2600 & 0.1967 & 0.0492 \\
& $\tau=1 / \pi$ & 36.4964 & 11.0742 & 6.5359 & 4.4783 & 3.8462 & 5.0839 & 20.3252 \\
\hline & & & & & & & &
\end{tabular}

Table 8. Summary of likelihood ratio test for stationarity of monthly FOREX sentiments.

\begin{tabular}{ccccc}
\hline Currency & $\begin{array}{c}\text { Observed value of chi } \\
\text { square statistics }\end{array}$ & Degrees of freedom & Table value of chi square & Decision \\
\hline EUR & 255.5811 & 56 & 98.0376 & Reject null hypothesis \\
GBP & 239.8553 & 53 & 92.3282 & Reject null hypothesis \\
SDR & 243.5784 & 51 & 87.3694 & Reject null hypothesis \\
YEN & 232.0151 & 55 & 94.3503 & Reject null hypothesis \\
ZAR & 229.5155 & 50 & 79.4900 & Reject null hypothesis \\
USD & 235.5667 & 50 & 79.4900 & Reject null hypothesis \\
\hline
\end{tabular}

$\pi_{1}=0.0694, \pi_{2}=0.0622, \pi_{3}=0.1326, \pi_{4}=0.2164, \pi_{5}=0.2381, \pi_{6}=0.2039, \pi_{7}=0.077$

We can make the following inference from the stationary probabilities.

$\pi_{1}=0.0694$ : The EURO exchange rate sentiment is gloomy for about $7 \%$ in a year, i.e., 0.84 months of the total 12 months.

$\pi_{3}=0.1326$ : The EURO exchange rate sentiment declines for about $13 \%$ in a year, 
i.e., 1.56 months of the total 12 months.

$\pi_{4}=0.2164$ : The EURO exchange rate sentiment is suggestive of normal activity for about $23 \%$ in a year, i.e., 2.76 months of the total 12 months.

The weekly expected recurrence time $\left\{\tau_{i i}, i=1, \cdots, 7\right\}$ for the seven states $S_{1}, \cdots, S_{7}$ is given by $\tau_{11}=14.41, \tau_{22}=16.08, \tau_{33}=7.54, \tau_{44}=4.62, \tau_{55}=4.20, \tau_{66}=4.90, \tau_{77}=12.99$.

This implies that the EURO currency bounced back to dread sentiment state $S_{1}$ once in every 14.41 months and the bearish sentiment state $S_{5}$ once in every 16.08 months and so on. The application of the likelihood ratio test statistic (11) for EURO gives $-2 \ln \Lambda=255.58$. Table 7 provides the summary of the stationary probabilities and expected recurrence times of monthly FOREX sentiments for all the six currencies considered. In Table 8, we provide the summary of the likelihood ratio tests for the stationarity of the monthly FOREX sentiment states.

\section{Discussion and Conclusion}

In this paper, we have revisited the analysis of exchange rate sentiments in six major global currencies during the year 2001 to 2012. The global currencies considered are Euro (EUR), Great Britain Pound (GBP), Special Drawing Rights (SDR), Japanese Yen (YEN), South African Rand (ZAR) and United States Dollar (USD). The data considered is for a 12 year period from January 2001 to December 2012. The weekly and monthly fluctuations in exchange rates are the key to the proposed analysis. The interdependence in the weekly and monthly average exchange rates is captured in terms of a Markov model. The state space of the finite dimensional Markov Chain is arrived at using a data driven classification rule. The states of the Markov model are ordinal in nature and are appropriately named based on the common perceptions witnessed in a stock market. The analysis of the data is carried out in two directions. In the first place, we consider the aggregate data from 2001 to 2012 and estimate the transition probability matrix for each of the six FOREX currencies considered. We derive the stationary probabilities of the seven exchange rate sentiments and estimate the expected recurrence time for the process to each of the seven states. The conclusions drawn from the weekly expected recurrence for the seven states $S_{1}, \cdots, S_{7}$ are as follows: The EUR, GBP, SDR and YEN currency sentiments took longer time to return to the bearish state $\left(S_{2}\right)$ during the period 2001 to 2012. The USD currency sentiment took approximately the same time to return to the dread $\left(S_{1}\right)$ and bearish $\left(S_{2}\right)$ sentiments. In the case of ZAR currency, the time to return to the dread sentiment was the highest. For the monthly expected recurrence times, the states dread $\left(S_{1}\right)$, bearish $\left(S_{2}\right)$ and greed sentiments $\left(S_{7}\right)$ witnessed longer time to return as compared to other sentiments for the currencies EUR, GBP and SDR. The dread sentiment indicated by state $S_{1}$ was the least visited state for YEN and USD while the states $S_{1}$ and $S_{2}$ for ZAR. Clearly, both the weekly and monthly FOREX sentiments turned out by highly speculative during the study period.

The second direction of the analysis is to examine the weekly and monthly exchange rate sentiments for each year of study from 2001 to 2012. This analysis is carried out by 
examining the stationarity of the estimated TPM for the weekly and monthly data. The analysis revealed that the pattern of transition from one sentiment state to another is altered from year to year.

\section{References}

[1] Clark, P.K. (1973) A Subordinated Stochastic Process Model with Finite Variance for Speculative Prices. Econometrica, 41, 135-156. https://doi.org/10.2307/1913889

[2] Engle, R.F. (1982) Autoregressive Conditional Heteroscedasticity with Estimates of the Variance of United Kingdom Inflation. Econometrica, 50, 987-1008.

https://doi.org/10.2307/1912773

[3] Bollerslev, T.A. (1987) A Conditional Heteroscedastic Time Series Model for Speculative Prices and Rates of Return. Review of Economics and Statistics, 69, 542-547.

https://doi.org/10.2307/1925546

[4] Jacquier, E., Polson, N.G. and Rossi, P.E. (2004) Bayesian Analysis of Stochastic Volatility Models with Fat-Tails and Correlated Errors. Journal of Econometrics, 122, 185-212. https://doi.org/10.1016/j.jeconom.2003.09.001

[5] Harvey, A.C. and Shephard, N. (1996) The Estimation of an Asymmetric Stochastic Volatility Model for Asset Returns. Journal of Business and Economic Statistics, 14, 429-434.

[6] Rebonato, R. (2005) Forward-Rate Volatilities and the Swaption Matrix: Why Neither Time-Homogeneity Nor Time Dependence Will Do. International Journal of Theoretical and Applied Finance, 9, 705-746. https://doi.org/10.1142/S0219024906003767

[7] White, R. and Rebonato, R. (2008) A Swaption Volatility Model Using Markov Regime Switching. The Journal of Computational Finance, 12, 79-114.

https://doi.org/10.21314/JCF.2008.182

[8] Hautsch, N. and Ou, Y. (2008) Discrete-Time Stochastic Volatility Models and MCMCBased Statistical Inference. Humboldt-Universitätzu Berlin, Germany, SFB 649 “Economic Risk" Discussion Paper 2008-063.

[9] Park, B.-Jo. (2008) Herd Behaviour and Volatility in Financial Markets. The 3rd International Conference on Asia-Pacific Financial Markets.

[10] Hahn, M. and Sass. J. (2009) Parameter Estimation in Continuous Time Markov Switching Models: A Semi-Continuous Markov Chain Monte Carlo Approach. Journal of Bayesian Analysis, 4, 63-68. https://doi.org/10.1214/09-BA402

[11] Beaupain, R., Giot, P. and Mikael, P. (2010) Volatility Regimes and Liquidity Co-Movements in Cap-Based Portfolios. Revue de 1 Association française de finance, 31, 55-79.

[12] Chun, C.Lo. and Konstantinos, S. (2011) Local Volatility Calibration with the Markov Chain Approximation. London Metropolitan Business School, Working Paper Series, No. 29.

[13] Kushner, H.J. (1990) Numerical Methods for Stochastic Control Problems in Continuous time. SIAM Journal of Control and Optimization, 28, 999-1048. https://doi.org/10.1137/0328056

[14] Sopipan, N., Pairote, S. and Bhusana, P. (2012) Forecasting Volatility of Gold Price Using Markov Regime Switching and Trading Strategy. Journal of Mathematical Finance, 2, 121-131. https://doi.org/10.4236/jmf.2012.21014

[15] Rao, K.S. and Moseki, K.K. (2009) A Probabilistic Approach to Categorise Equity Index Volatility. Management Dynamics, 18, 22-34.

[16] Rao, K.S. and Moseki, K.K. (2011) Analyzing Volatility in Equity Indices-A Markov Ap- 
proach for Botswana Domestic Company Indices. South African Journal of Industrial Engineering, 22, 83-98.

[17] Rao, K.S.M. and Anjana, R. (2014) Exchange Rate Market Sentiment Analysis of Major Global Currencies. Open Journal of Statistics, 4, 49-69.

https://doi.org/10.4236/ojs.2014.41006

[18] Bhat, N.U. (1972) Elements of Applied Stochastic Processes. John Wiley \& Sons Inc., New York.

[19] Isaacson, D.L. and Madsen, R.W. (1976) Markov Chains Theory and Applications. John Wiley \& Sons Inc., London.

Submit or recommend next manuscript to SCIRP and we will provide best service for you:

Accepting pre-submission inquiries through Email, Facebook, LinkedIn, Twitter, etc.

A wide selection of journals (inclusive of 9 subjects, more than 200 journals)

Providing 24-hour high-quality service

User-friendly online submission system

Fair and swift peer-review system

Efficient typesetting and proofreading procedure

Display of the result of downloads and visits, as well as the number of cited articles

Maximum dissemination of your research work

Submit your manuscript at: http://papersubmission.scirp.org/

Or contact ojs@scirp.org 